\title{
A STUDY TO FURTHER UNDERSTAND THE LINK BETWEEN IMMERSION AND FLOW
}

\author{
Ehm Kannegieser ${ }^{1}$, Daniel Atorf ${ }^{1}$ and Josua Meier $^{2}$ \\ ${ }^{I}$ Fraunhofer Institute of Optronics, System Technologies and Image Exploitation \\ Fraunhoferstraße. 1, 76131, Karlsruhe, Germany \\ ${ }^{2}$ Karlsruhe Institute of Technology, Kaiserstraße. 12, 76131, Karlsruhe, Germany
}

\begin{abstract}
Flow and Immersion are states of extreme concentration on an activity. For Serious Games, games which focus on achieving learning effects in players, high Flow and Immersion during gameplay can help improve these learning effects. Both Flow and Immersion are currently only measured using questionnaires, which is both delayed and subjective. This work introduces a study which aims to further the understanding of how Flow and Immersion are linked and to ease future work towards a new measurement method using physiological data.
\end{abstract}

\section{KEYWORDS}

Flow, Immersion, Combined Model, Correlation, Measurement, Physiological

\section{INTRODUCTION}

The design of Serious Games still presents an active field of research. Especially important are techniques to increase the learning rate of these games. Previous studies find in their research that high focus helps to transmit learning material through Serious Games (Deci and Ryan, 1985; Krapp, 2009). Two terms used to measure high focus are Flow and Immersion. These terms describe states of great focus on an activity or game. They are currently measured using questionnaires (Nordin, Denisova and Cairns, 2014), which have a range of problems. A participant cannot fill out a questionnaire without interrupting the concentration state, meaning there must be a delay when gathering data. Additionally, questionnaires are inherently subjective, adding additional bias to the measured results. The solution to this problem would be to measure changes in the player's body and derive Flow and Immersion from that. This work tries to present a step towards this solution. In order to better understand how Flow and Immersion interact, a combined model is established. Afterwards, based on previous work (Atorf et al., 2016; Kannegieser et al. 2018), a study is presented, which attempts to validate the model and link it with physiological measurements taken from players.

\section{PREVIOUS WORK}

Flow was first described by Csikszentmihalyi as the state of the optimal experience of an activity (Csikszentmihalyi, 1991). When entering a state of Flow, even taxing activities like work no longer feel taxing, but rather feel enjoyable. However, the Flow state cannot be achieved for every activity. Csikszentmihalyi bases flow on the model of extrinsic and intrinsic motivation. Only intrinsically motivated actions, which are not motivated by external factors, can reach the Flow state. Flow is reachable when the challenge presented by such an intrinsically motivated action is balanced with the skill of the person performing the task. All this makes Flow an interesting point of research in regard to games, as playing games is usually intrinsically motivated. Flow is mapped to games in the GameFlow questionnaire (Sweetser et al., 2005). 
There exist two concurrent definitions of Immersion. The first definition is called presence-based Immersion and refers to the feeling of being physically present in a virtual location. The second definition is known as engagement-based Immersion. It defines Immersion based on the strength of a player's interaction with the game. The model given by Cairns et al. in their series of papers (Cairns et al., 2006; Jenett et al., 2008), defines Immersion as a hierarchical structure, with different barriers of entry. Figure 1 left shows this hierarchy. The lowest level, Engagement, is reached by interacting with the game and spending time with it. Engrossment is reached by becoming emotionally involved with the game. During this state, feelings of temporal and spatial dissociation are starting to appear. The final state, Total Immersion, is reached by players having their feelings completely focused on the game. Cheng et al. improve upon this hierarchical model by adding dimensions to the three levels of the hierarchy (Cheng et al., 2015). The Engagement level is split into the three dimensions: Attraction, Time Investment and Usability. The second level, Engrossment, is split into Emotional Attachment, which refers to attachment to the game itself, and Decreased Perceptions. Finally, Total Immersion is defined by the terms Presence and Empathy.

Flow and Immersion share many similarities. Both have similar effects, such as decreased perceptions of both time and the environment, and refer to a state of focus. Georgiou and Kyza even take the empathy dimension in the immersion model by Cheng et al. and replace it with Flow (Georgiou and Kyza, 2017). There are two main differences between the two: First, Flow does not define an emotional component, while Immersion is focused heavily on the emotional attachment of players to the game. Second, while Flow refers to a final state of complete concentration, Immersion refers to a range of experiences, ranging from minimal engagement to complete focus on the game. This is reflected in how those two psychological states are described in their introduction. Csikszentmihalyi describes Flow as the "optimal experience of an activity", while Cairns et al. describe Immersion as the "sub-optimal experience of an activity".

\section{MODEL}

The model used in the study is based on the Flow model presented by Csikszentmihalyi (Csikszentmihalyi, 1991) and the Immersion model by Cheng et al. (Cheng et al., 2015), which itself is a refinement of the hierarchical model presented by Cairns et al. Flow, as the optimal experience of an action, is considered the highest point in the Immersion hierarchy, which implies that Total Immersion and Flow are regularly experienced together. Figure 1 center presents the Immersion hierarchy imposed on top of the three-channel model by Csikszentmihalyi. As Immersion grows, the possibility to reach the Flow state increases. It must be noted that the diagram is only meant to be a qualitative visualization, as Immersion is not dependent on the challenge/skill balance.
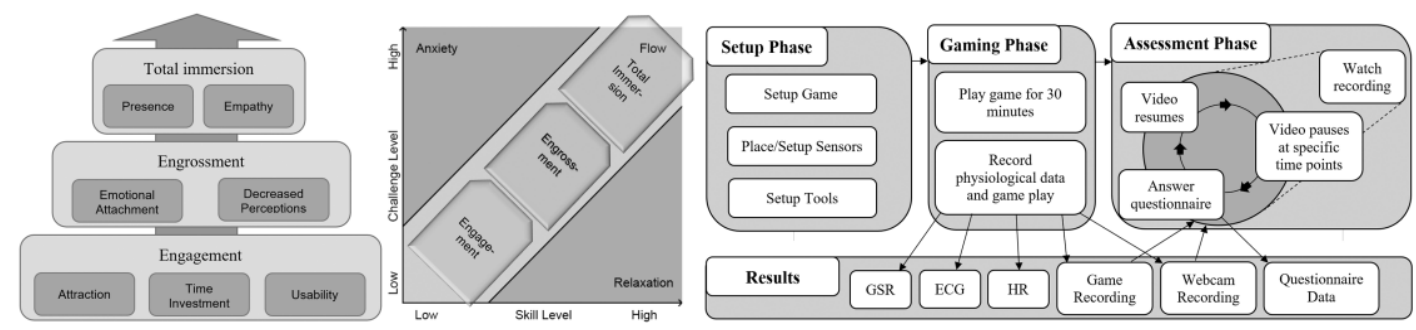

Figure 1. Left: Levels of the Immersion model by Cairns et al., with dimensions for each level by Cheng et al. (Cairns et al., 2006; Cheng et al., 2015). Center: Hierarchical Immersion model on top of the three-channel Flow model by Csikszentmihalyi (Csikszentmihalyi, 1991). Right: Stages of the study described in section 4

\section{EXPERIMENT}

The study was designed to both gather data to link physiological measurements with Flow and Immersion, as well as validate the Flow/Immersion model presented in section 3. 40 participants took part in the study. Participants were self-selected. This method was chosen to help observe higher levels of Immersion and 
Flow. The study is split into three phases. During the Setup Phase, a game is selected. Free choice of game makes finding links between physiological measurements harder, but was chosen to help participants reach the Flow state more easily. During the Gaming Phase, participants play the game for 30 minutes. For 30 minutes, physiological measurements are taken. The physiological measurements used in this study were used due to being non-intrusive and not hindering the immersion of players. Galvanic skin response, Electrocardiography, gaze tracking and web cam footage for emotion analysis were used. A facial EMG would be more precise for analyzing displayed emotions, however, placing electrodes on the face of the player would distract from the gameplay experience and make it harder to reach the flow state. For the same reasons, EEG measurement was not chosen for the study. After the Gaming Phase has concluded, participants enter the Assessment Phase and watch their previous gaming session, while answering questionnaires about Immersion and Flow periodically. This setup was chosen to get more exact results and because it does not interrupt the Flow experience. Three questionnaires are used during the study. The first questionnaire used is the Immersion questionnaire described by Cheng et al (Cheng et al., 2015). As the questionnaire was too long to be measured multiple times without worsening the results, it was split into an Immersive Tendency questionnaire asked at the beginning of playback and an iterative questionnaire asked every three minutes during playback. For Flow, the Flow Short Scale questionnaire by Rheinberg et al. was used (Rheinberg et al., 2003). It was originally designed for being used multiple times in a row, making it perfect for this iterative approach. During playback, it is asked every six minutes. The final questionnaire used is the Game Experience Questionnaire (IJsselsteijn, de Kort and Poels, 2013). It measures a more general set of questions and is asked once after playback is over. Figure 1 right shows the three phases of the experiment as well as the activities of each phase.

\section{PRELIMINARY RESULTS}

The first step of the analysis is to check for correlations between Flow and Immersion. The results from both the Flow and Immersion questionnaires do not follow a normal distribution. For that reason, Spearman correlation was chosen. The correlation analysis found a strong correlation between all three levels of Immersion and Flow. The strongest correlation was found between Engagement and Flow $(\mathrm{R}=0.69$, $\mathrm{p}=8.536 \mathrm{e}-30$ ), which makes sense, knowing that Flow encompasses all features making up Engagement. The second strongest correlation exists between Total Immersion and Flow $(R=0.652, p=1.91 e-25)$. This is caused by the fact that players who played games without clear avatars, such as strategy games, found it difficult to emphasize with their avatar in the game, leading to reduced Total Immersion. The least correlated level of the three is Engrossment $(\mathrm{R}=0.56, \mathrm{p}=1.829 \mathrm{e}-18)$, which can be explained as Engrossment puts strong emphasis on emotional attachment of the player to the game, something Flow does not elicit. All three show strong correlation to Flow, meaning the relation between these two psychological states explained in section 3 is likely. Table 1 displays the Rho coefficients for all states. Direct correlation between normalized physiological data and answers of the Flow/Immersion questionnaires show no meaningful correlation. The direct correlation results are shown in table 1 . This means a more advanced method has to be employed. The first advanced method tried was using expert rules and fuzzy logic to create rules and activation methods based on an approach used by Goshvarpour et al. (Goshvarpour et al., 2017). Using this approach did not lead to meaningful results either, as it was difficult to create useful rules from the raw data and direct correlation results between physiological measurements and Flow/Immersion.

Table 1. Spearman Rho correlation coefficients for Flow/Immersion and physiological measurements

\begin{tabular}{|c|c|c|c|c|c|c|c|}
\hline & Flow & Engagement & Engrossment & $\begin{array}{l}\text { Total } \\
\text { Immersion }\end{array}$ & GSR & Heartrate & $\begin{array}{l}\text { Restpoints } \\
\text { Per } \\
\text { minute }\end{array}$ \\
\hline Flow & 1 & 0.69 & 0.57 & 0.65 & -0.02 & -0.03 & -0.07 \\
\hline Engagement & 0.69 & 1 & 0.45 & 0.58 & 0.01 & -0.08 & -0.02 \\
\hline $\begin{array}{l}\text { Engrossment } \\
\text { Total }\end{array}$ & 0.57 & 0.45 & 1 & 0.62 & -0.04 & -0.09 & 0.05 \\
\hline Immersion & 0.65 & 0.58 & 0.62 & 1 & -0.15 & 0 & 0.06 \\
\hline
\end{tabular}




\section{CONCLUSION}

With this work, an attempt was made to link physiological reactions in players to the states of Flow and Immersion. Furthermore, a second aim was to get a clearer understanding of how Flow and Immersion are linked, and present a unified model. In order to reach these goals, a study was performed and the results evaluated. As far as the link between Immersion and Flow is concerned, the results back up the model presented in section 3. The data shows strong correlation between high Flow and high Immersion for all three Immersion levels, which would imply Flow to be situated at the highest point of the Immersion hierarchy, along with total Immersion. Results are less clear for the link between physiological measurements and the Flow/Immersion model. Direct correlation did not find any connection between the recorded data. This also made creating an advanced rule-based system, as explained in chapter 4, difficult to achieve. Future research will be aimed at further analyzing the connection between physiological measurements and Flow with other more advanced techniques.

\section{REFERENCES}

Atorf, D., L. Hensler, and E. Kannegieser (2016). "Towards a concept on measuring the Flow state during gameplay of serious games". In: European Conference on Games Based Learning (ECGBL). ECGBL 2016. Paisley, Scotland, pp. 955-959. isbn: 978-1-911218-09-8. url: http://publica.fraunhofer.de/documents/N-438328.html.

Kannegieser E., Daniel Atorf and Josua Meier (2018). "Surveying games with a combined model of Immersion and Flow". In: MCCSIS 2018 Multi Conference on Computer Science and Information Systems, Game and Entertainment Technologies 2018.

Cairns, Paul (2006). "Quantifying the experience of immersion in games".

Cheng, M.-T., H.-C. She, and L.A. Annetta (June 2015). "Game Immersion Experience: Its Hierarchical Structure and Impact on Game-based Science Learning”. In: J. Comp. Assist. Learn. 31.3, pp. 232-253. issn: 0266-4909. doi: 10.1111/jcal.12066. url: http: //dx.doi.org/10.1111/jcal.12066.

Csikszentmihalyi, Mihaly (Mar. 1991). Flow: The Psychology of Optimal Experience. New York, NY: Harper Perennial. isbn: 0060920432. url: http : / / www . amazon . com / gp / product/0060920432/ref=si3_rdr_bb_product/1044616565-4570345.

Deci, Edward and Richard Ryan (Jan. 1985). Intrinsic Motivation and Self-Determination in Human Behavior. Vol. 3.

Georgiou, Yiannis and Eleni A. Kyza (Feb. 2017). "The Development and Validation of the ARI Questionnaire". In: Int. J. Hum.-Comput. Stud. 98.C, pp. 24-37. issn: 1071-5819. doi: 10.1016/j.ijhcs.2016.09.014. url: https://doi.org/10.1016/j.ijhcs.2016.09.

Goshvarpour, Atefeh, Ataollah Abbasi, and Ateke Goshvarpour (2017). “An accurate emotion recognition system using ECG and GSR signals and matching pursuit method". In: Biomedical Journal 40.6, pp. 355-368. issn: 2319-4170. doi: https://doi.org/10. 1016/j.bj.2017.11.001. url: http://www.sciencedirect.com/science/article/pii/ S2319417016301056.

IJsselsteijn, W.A. et al. (2008). "Measuring the experience of digital game enjoyment". English. In: Proceedings of Measuring Behavior 2008, 6th International Conference on Methods and Techniques in Behavioral Research, August 26-29, 2008, Maastricht. Ed. by A.J. Spink et al. Noldus, pp. 88-89. isbn: 978-90-74821-81-0.

Jennett, Charlene et al. (Sept. 2008). "Measuring and Defining the Experience of Immersion in Games". In: Int. J. Hum.-Comput. Stud. 66.9, pp. 641-661. issn: 1071-5819. doi: 10. 1016/j.ijhcs.2008.04.004. url: http://dx.doi.org/10.1016/j.ijhcs.2008.04.004.

Krapp, Andreas, Ulrich Schiefele, and Inge Schreyer (2009). Metaanalyse des Zusammenhangs von Interesse und schulischer Leistung. postprint

Nordin, A. Imran, Alena Denisova, and Paul Cairns (Oct. 2014). "Too Many Questionnaires: Measuring Player Experience Whilst Playing Digital Games".

Rheinberg, F., R. Vollmeyer, and S. Engeser (2003). “Die Erfassung des Flow-Erlebens". In: Diagnostik von Motivation und Selbstkonzept. Göttingen: Hogrefe, pp. 261-279.

Sweetser, Penelope and Peta Wyeth (July 2005). "GameFlow: A Model for Evaluating Player Enjoyment in Games". In: Comput. Entertain. 3.3, pp. 3-3. issn: 1544-3574. doi: 10.1145/1077246.1077253. url: http://doi.acm.org/10.1145/1077246.1077253. 